\title{
Numerical simulation of heat transfer in packed beds by two population thermal lattice Boltzmann method
}

\author{
ROBERT STRAKA ${ }^{\mathrm{a}}$ \\ Department of Heat Engineering and Environment Protection, Faculty of Metals Engineering and Industrial Computer \\ Science, AGH University of Science and Technology, Aleja Mickiewicza 30, 30-059 Cracow, Poland
}

Received 2 December 2014, Accepted 11 April 2015

\begin{abstract}
The Lattice Boltzmann Method (LBM) was used for simulation of a gas flow and conjugate heat transfer in fixed packed beds with solid particles inside. D2Q9 version of the Factorized Central Moment (FCMLBM) is used for a gas flow computation. Heat transfer in the bed is described by second distribution function set and multiple relaxation time lattice Boltzmann method (DDF MRT LBM) is used. Numerical solver is implemented using NVIDIA CUDA framework and comparison of performance on different GPUs is presented as well. Validation of the code is done using experimentally measured values of temperatures in fixed rock bed in a high-temperature thermal storage experiment. Next, the rate of heat transfer in a large scale fixed bed is numerically investigated, together with description of the bed's thermal characteristics for two different mass flow rates of hot air.
\end{abstract}

Key words: Lattice Boltzmann method / packed bed / conjugate heat transfer

\section{Introduction}

Description of a conjugate heat transfer in packed beds is of big interests in various branches of process industry (metallurgy, chemical, food processing, etc.). Our aim is to describe heat and fluid flow in shaft a furnace i.e. metallurgical device used for rock melting. Models of shaft furnaces are very rare in literature [1-3] (all those are only 1D), it is due to the complicated load (randomly packed, nonspherical particles) geometry of such furnaces. Multiple granular materials resulting in variable porosity make full numerical description of the problem problematic. Complex mesh generation when using standard numerical methods like FDM, FEM or FVM in order to solve NavierStokes and Fourier-Kirchhoff equations is inevitable. The novel approach of solving those equations based on the discrete version [4] of the Boltzmann transport equation is called the lattice Boltzmann method (LBM), it is proven to be suitable for problems with complex geometry [5], thermal versions of LBM (TLBM) were successfully used in different heat transfer problems [6-8] and due to application and optimization of code on GPUs $[6,8,9]$, this method could deliver high performance numerical codes for the heat transfer and fluid flow. During last decades, several approaches were developed, those are single relaxation time (SRT) [4], multiple relaxation time (MRT) [10],

\footnotetext{
${ }^{a}$ Corresponding author: straka@metal.agh.edu.pl
}

entropic lattice Boltzmann method (ELBM) [11] and cascaded lattice Boltzmann method (CLBM) [12]. All these methods have their advantages and drawbacks which can be stated by the observation that the higher the stability of the approach is the higher the complexity and resources demands are. Extensive review works on the state of the art LBM methods $[5,13]$ exist together with several books on this topic $[4,14-16]$.

Thermal LBMs can be generally divided to the three groups. Multiple speed methods (one population model with extended set of characteristic velocities) [17], double distribution methods (treating temperature as a passive scalar) [18] and hybrid methods (using FDM, FEM or FVM to solve energy equation) [7]. Simple double distribution method based on MRT was used, more advanced double distribution models are described in references [19-21].

LBM with its stability, accuracy and robustness even for highly complicated meshes is very attractive when considering basic problems in metallurgy which are based on heat and mass transfer together with fluid flow in irregular and porous structures like fixed or moving beds with changing geometry of particles (e.g. due to combustion). Another attractive field of possible TLBM application in metallurgy is numerical study of continuous steel casting [22]. In material engineering, TLBM can be applied in numerical investigation of heat transfer efficiency of double finned radially twisted heat exchanger tubes with 


\section{Nomenclature}

\begin{tabular}{|c|c|c|}
\hline $\mathbf{c}_{i}$ & & $i$ th characteristic lattice velocity \\
\hline$c$ & & Characteristic lattice velocity magnitude \\
\hline$c_{s}$ & $\mathrm{~m} \cdot \mathrm{s}^{-1}$ & Speed of sound \\
\hline$D$ & $\mathrm{~m}$ & Diameter of the domain \\
\hline$d$ & $\mathrm{~m}$ & Particle diameter \\
\hline $\mathbf{e}_{i}$ & & $i$ th unit characteristic lattice velocity \\
\hline$f_{i}, g_{i}$ & & $i$ th distribution function \\
\hline$\dot{G}$ & $\mathrm{~kg} \cdot \mathrm{m}^{-2} \cdot \mathrm{s}^{-1}$ & Cross-sectional mass flow rate \\
\hline$H$ & $\mathrm{~m}$ & Height of the domain \\
\hline $\mathbf{K}$ & & Moment matrix of CLBM \\
\hline $\mathbf{k}$ & & Moments of CLBM \\
\hline$L$ & $\mathrm{~m}$ & Characteristic length \\
\hline M & & Moment matrix of MRT \\
\hline$\dot{M}$ & $\mathrm{~kg} \cdot \mathrm{s}^{-1}$ & Air mass flow rate \\
\hline $\mathbf{m}$ & & Moments of MRT \\
\hline$N$ & & Number of lattice sites \\
\hline$p$ & $\mathrm{~kg} \cdot \mathrm{m}^{-1} \cdot \mathrm{s}^{-2}$ & Pressure \\
\hline $\mathrm{Pe}$ & & Peclet number \\
\hline $\operatorname{Pr}$ & & Prandtl number \\
\hline $\operatorname{Re}$ & & Reynolds number \\
\hline S & & Relaxation matrix \\
\hline$T$ & $\mathrm{~K}$ & Temperature \\
\hline$t$ & $\mathrm{~s}$ & Time \\
\hline$\Delta t$ & s & Time step \\
\hline $\mathbf{U}$ & $\mathrm{m} \cdot \mathrm{s}^{-1}$ & Characteristic velocity \\
\hline $\mathbf{u}$ & $\mathrm{m} \cdot \mathrm{s}^{-1}$ & Velocity vector \\
\hline$w_{i}$ & & $i$ th Weight factor \\
\hline $\mathbf{x}$ & $\mathrm{m}$ & Spatial position vector \\
\hline & & Greek letters \\
\hline$\alpha$ & $\mathrm{m}^{2} \cdot \mathrm{s}^{-1}$ & Thermal diffusivity \\
\hline$\delta$ & & Lattice spatial (temporal) step \\
\hline$\epsilon$ & & Porosity \\
\hline$\kappa$ & & Central moment \\
\hline$\Lambda$ & & Magic number \\
\hline$\nu$ & $\mathrm{m}^{2} \cdot \mathrm{s}^{-1}$ & Kinematic viscosity \\
\hline$\pi$ & & Raw moment \\
\hline$\rho$ & $\mathrm{kg} \cdot \mathrm{m}^{-3}$ & Density \\
\hline$\tau$ & & Relaxation time \\
\hline & & Subscripts \\
\hline 0 & & Initial \\
\hline $\mathrm{b}$ & & Boundary \\
\hline $\mathrm{f}$ & & Fluid \\
\hline in & & Inlet \\
\hline s & & Solid \\
\hline$x$ & & Spatial or in $x$ axis direction \\
\hline$y$ & & In $y$ axis direction \\
\hline & & Superscripts \\
\hline$*$ & & Post-collisional \\
\hline+ & & Dimensionless \\
\hline at & & Attractor \\
\hline eq & & Equilibrium \\
\hline & & Abbreviations \\
\hline LBM & & Lattice Boltzmann Method \\
\hline FCM & & Factorized Central Moment \\
\hline CLBM & & Cascaded Lattice Boltzmann Method \\
\hline DDF & & Double Distribution Function \\
\hline CUDA & & Compute Unified Device Architecture \\
\hline GPU & & Graphic Processing Unit \\
\hline FDM & & Finite Difference Method \\
\hline FEM & & Finite Element Method \\
\hline FVM & & Finite Volume Method \\
\hline TLBM & & Thermal Lattice Boltzmann Method \\
\hline SRT & & Single Relaxation Time \\
\hline MRT & & Multiple Relaxation Time \\
\hline ELBM & & Entropic Lattice Boltzmann Method \\
\hline MLUPS & & Million Lattice Updates Per Second \\
\hline
\end{tabular}

complicated fin geometry [23, 24] without need for the complicated numerical meshing which is essential in classical numerical methods to achieve convergence and accuracy. However, in this paper we focus on the conjugate heat transfer in fixed packed beds which represents load in shaft furnaces. The paper presents double distribution function TLBM based on CLBM for fluid flow and MRT for heat transfer, validation of the model for the case of thermal storage experiment and thermal characteristics of fixed bed with differently sized particles.

\section{Lattice Boltzmann method for two-dimensional heat transfer and fluid flow}

The Lattice Boltzmann Method is derived from Boltzmann transport equation through spatial and velocity space discretization. The fluid is described by "virtual" particles living on the regular lattice, colliding and streaming along the fixed characteristic directions (determined by characteristic velocities set). Macroscopic quantities are obtained from moments of particles' distribution functions. General notation used in LBM community for d-dimensional model with q characteristic velocities is DdQq, later on we will focus on two-dimensional model with nine and five characteristic speeds i.e. D2Q9 for fluid flow and D2Q5 for heat transfer models.

The force-free discretized lattice Boltzmann equation with Bhatnagar-Gross-Krook collision operator [25] reads

$$
\begin{array}{r}
f_{i}\left(\mathbf{x}_{j}+\mathbf{c}_{i} \delta_{t}, t+\delta_{t}\right)=f_{i}\left(\mathbf{x}_{j}, t\right)-\frac{1}{\tau}\left(f_{i}\left(\mathbf{x}_{j}, t\right)\right. \\
\left.-f_{i}^{\mathrm{eq}}\left(\mathbf{x}_{j}, t\right)\right)=f_{i}^{*}\left(\mathbf{x}_{j}, t\right)
\end{array}
$$

where $f_{i}$ is $i$ th distribution function associated with $i$ th characteristic velocity $\mathbf{c}_{i}=c \mathbf{e}_{\mathbf{i}}$ with $\mathbf{e}_{i}$ being discrete velocity and $c=\delta_{x} / \delta_{t}$ lattice speed. $f_{i}^{\text {eq }}$ is equilibrium distribution function, $f_{i}^{*}$ denotes post-collision state, $\delta_{x}$ and $\delta_{t}$ are spatial and temporal step, $\tau$ is relaxation time. Through Chapmann-Enskog expansion [26] or Hilbert expansion [27] it can be shown that Navier-Stokes equations (or the Fourier-Kirchhoff equation) can be recovered. Relaxation time is related to the viscosity or heat diffusivity and the relation can be obtained through the expansions mentioned above. The problem solved with LBM is usually first nondimensionalized and then rescaled into so called lattice units. Spatial and temporal step is usually set to be equal to one $\left(\delta_{t}=\delta_{x}=c=1\right)$ and from now on we will use these units in all equations related to LBE.

Algorithm of solving the LBM consists of just few steps. First of all every lattice site is initialized to it's equilibrium by computing $f_{i}^{\text {eq }}$ from given density ${ }^{1} \rho^{+}$ and velocity $\mathbf{u}^{+}=\left(u_{x}^{+}, u_{y}^{+}\right)^{\mathrm{T}}$, then $f_{i}$ are "streamed" along appropriate characteristic velocity vector (so called

\footnotetext{
${ }^{1}$ Note that these parameters are in lattice units i.e. designated with "+" superscript, which will be dropped in the next text, except where needed to prevent confusion.
} 

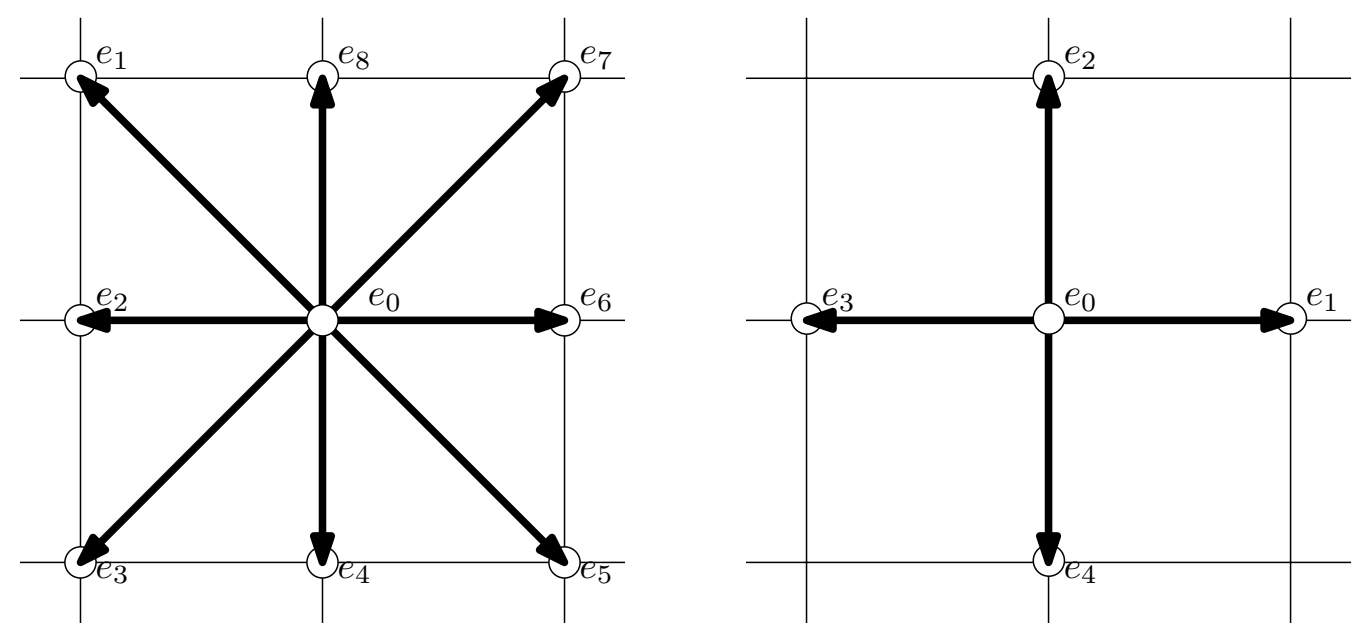

Fig. 1. Characteristic velocities for D2Q9 (left) and D2Q5 (right) models.

streaming step), boundary conditions are set and new macroscopic quantities are computed from $f_{i}$, after that collision is performed i.e. relaxation towards equilibrium in case of BGK operator (so called collision step). Last three steps are repeated until desired time or steady state is achieved.

The last ingredients here are the equation for the equilibrium distribution functions and relation between $\tau$ and $\nu$. Equilibrium distribution functions are given by Taylor expansion of Maxwell's distribution function [15]

$$
f_{i}^{\mathrm{eq}}=\rho w_{i}\left(1+\frac{\mathbf{u} \cdot \mathbf{e}_{i}}{c_{s}^{2}}+\frac{\left(\mathbf{u} \cdot \mathbf{e}_{i}\right)^{2}}{2 c_{s}^{4}}-\frac{\mathbf{u} \cdot \mathbf{u}}{2 c_{s}^{2}}\right)
$$

where $w_{i}$ and $c_{s}$ are weight factors and lattice speed of sound, they depend on the used lattice and on the set of characteristic velocities. Relaxation factor is related to fluid viscosity by

$$
\tau=\frac{\nu}{c_{s}^{2}}+\frac{1}{2}
$$

Macroscopic density and velocities are given by following moments of $f_{i}$ :

$$
\begin{gathered}
\rho=\sum_{i} f_{i} \\
\rho \mathbf{u}=\sum_{i} f_{i} \mathbf{e}_{i}
\end{gathered}
$$

and pressure can be obtained by lattice equation of state

$$
p=\rho c_{s}^{2}
$$

\section{$2.1 \mathrm{D} 2 \mathrm{Q} 9$ and $\mathrm{D} 2 \mathrm{Q} 5$ model}

In our numerical investigations the D2Q9 model was used for fluid flow and D2Q5 for heat transfer. Set of characteristic velocities together with weighting factors and speeds of sound is given below. Nine discrete velocities of D2Q9 model are given by the set

$$
\left[e_{0}, \ldots, e_{8}\right]=\left(\begin{array}{ccccccccc}
0 & 1 & -1 & -1 & 0 & 1 & 1 & 1 & 0 \\
0 & -1 & 0 & -1 & -1 & -1 & 0 & 1 & 1
\end{array}\right)
$$

with the set of weights

$$
\left\{w_{0}, \ldots, w_{8}\right\}=\left\{\frac{4}{9}, \frac{1}{36}, \frac{1}{9}, \frac{1}{36}, \frac{1}{9}, \frac{1}{36}, \frac{1}{9}, \frac{1}{36}, \frac{1}{9}\right\}
$$

and the square of sound speed is equal to $c_{s}^{2}=1 / 3$. Five discrete velocities of D2Q5 model are given by the above set without diagonal vectors i.e.

$$
\left\{e_{0}, \ldots, e_{4}\right\}=\{(0,0) ;(1,0) ;(0,1) ;(-1,0) ;(0,-1)\}
$$

with the set of weights

$$
\left\{w_{0}, \ldots, w_{4}\right\}=\left\{\frac{1}{3}, \frac{1}{6}, \frac{1}{6}, \frac{1}{6}, \frac{1}{6}\right\}
$$

and again the square of sound speed is equal to $c_{s}^{2}=1 / 3$. Equilibrium distribution function is defined as

$$
g_{i}^{\mathrm{eq}}=T w_{i}\left(1+\frac{\mathbf{u} \cdot \mathbf{e}_{i}}{c_{s}^{2}}\right)
$$

Characteristic velocities within lattice site are depicted in Figure 1. Performing Chapmann-Enskog expansion, it can be shown that for the case of the BGK D2Q9 [28] and D2Q5 models, following Navier-Stokes and FourierKirchhoff equations can be recovered

$$
\frac{\partial u_{x}}{\partial x}+\frac{\partial u_{y}}{\partial y}=\mathrm{O}\left(\mathrm{Ma}^{2}\right)
$$

$$
\begin{aligned}
\frac{\partial u_{x}}{\partial t}+\frac{\partial u_{x}^{2}}{\partial x}+\frac{\partial u_{x} u_{y}}{\partial y}= & -\frac{\partial p}{\partial x}+\frac{1}{\operatorname{Re}}\left[\frac{\partial}{\partial x}\left(\frac{\partial u_{x}}{\partial x}\right)\right. \\
& \left.+\frac{\partial}{\partial y}\left(\frac{\partial u_{x}}{\partial y}\right)\right]+\mathrm{O}\left(\mathrm{Ma}^{3}\right) \\
\frac{\partial u_{y}}{\partial t}+\frac{\partial u_{y} u_{x}}{\partial x}+\frac{\partial u_{y}^{2}}{\partial y}= & -\frac{\partial p}{\partial y}+\frac{1}{\operatorname{Re}}\left[\frac{\partial}{\partial x}\left(\frac{\partial u_{y}}{\partial x}\right)\right. \\
& \left.+\frac{\partial}{\partial y}\left(\frac{\partial u_{y}}{\partial y}\right)\right]+\mathrm{O}\left(\mathrm{Ma}^{3}\right) \\
\frac{\partial T}{\partial t}+u_{x} \frac{\partial T}{\partial x}+u_{y} \frac{\partial T}{\partial y}= & \alpha\left(\frac{\partial^{2} T}{\partial x^{2}}+\frac{\partial^{2} T}{\partial y^{2}}\right)+\mathrm{O}\left(\Delta t^{2}\right)
\end{aligned}
$$


where Mach number is defined as $\mathrm{Ma}=|\mathbf{u}| / c_{s}$ and $\Delta t$ is the time step in physical units $\left(\Delta t=\left|\mathbf{U}^{+}\right| L /\left(|\mathbf{U}| L^{+}\right)\right.$ where "+" denotes lattice units and $\mathbf{U}$ and $L$ are characteristic velocity and length respectively).

\subsection{Cascaded lattice Boltzmann method}

To improve the stability of a SRT BGK model, advanced MRT models were introduced and successfully used till present time. However Geier et al. [12] realized that stability of MRT models can be improved drastically when the Galilean invariance of MRT is restored. This is done by performing the collision step in momentum space with central moments used in BGK operator instead of raw ones. The main ideas of the CLBM will be briefly described in this section.

Basic idea of MRT is performing collision step in momentum space i.e. distribution functions vector $\mathbf{f}=$ $\left(f_{0}, \ldots, f_{q-1}\right)^{\mathrm{T}}$ is transformed by matrix $\mathbf{M}$ into momentum space $\left(m_{0}, \ldots, m_{q-1}\right)^{\mathrm{T}}=\mathbf{m}=$ Mf. Moments are equilibrated towards their appropriate momentum equilibria $\mathbf{m}^{\mathrm{eq}}$ using diagonal matrix $\mathbf{S}=$ $\operatorname{diag}\left(1 / \tau_{0}, \ldots, 1 / \tau_{q-1}\right)$ i.e. with different relaxation times (MRT reduces to SRT when all $\tau_{i}$ are equal) and transformed back using inverse of $\mathbf{M}$. The MRT LBE therefore reads

$f_{i}\left(\mathbf{x}_{j}+\mathbf{e}_{i}, t+1\right)=f_{i}\left(\mathbf{x}_{j}, t\right)-\mathbf{M}^{-1} \mathbf{S}\left(m_{i}\left(\mathbf{x}_{j}, t\right)-m_{i}^{\mathrm{eq}}\left(\mathbf{x}_{j}, t\right)\right)$

We use transformation matrix given in [12] and denote it $\mathbf{K}$

$$
\mathbf{K}=\left(\begin{array}{ccccccccc}
1 & 0 & 0 & -4 & 0 & 0 & 0 & 0 & 4 \\
1 & -1 & 1 & 2 & 0 & 1 & -1 & 1 & 1 \\
1 & -1 & 0 & -1 & 1 & 0 & 0 & -2 & -2 \\
1 & -1 & -1 & 2 & 0 & -1 & 1 & 1 & 1 \\
1 & 0 & -1 & -1 & -1 & 0 & -2 & 0 & -2 \\
1 & 1 & -1 & 2 & 0 & 1 & 1 & -1 & 1 \\
1 & 1 & 0 & -1 & 1 & 0 & 0 & 2 & -2 \\
1 & 1 & 1 & 2 & 0 & -1 & -1 & -1 & 1 \\
1 & 0 & 1 & -1 & -1 & 0 & 2 & 0 & -2
\end{array}\right)
$$

next we define raw moments of $f_{i}$ as

$$
\pi_{x^{m} y^{n}}=\sum_{i} f_{i} e_{i, x}^{m} e_{i, y}^{n}
$$

and central moments of $f_{i}$ as

$$
\kappa_{x^{m} y^{n}}=\sum_{i} f_{i}\left(e_{i, x}-u_{x}\right)^{m}\left(e_{i, y}-u_{y}\right)^{n}
$$

where $m, n \in \mathcal{Z}$ and $m+n$ is the order of the moment. The attractors for collision step are defined as

$$
f^{\text {at }}=f+\mathbf{K} \cdot \mathbf{k}
$$

and central moments are computed for both sides of the Equation (3) i.e. we multiply it by $\left(e_{i, x}-u_{x}\right)^{m}\left(e_{i, y}-u_{y}\right)^{n}$ and take the sum over $i$. In result we obtain system of six equations (first three moments are conserved quantities i.e. collisional invariants and thus not involved here) for $k_{3}, \ldots, k_{8}$

$$
\begin{gathered}
\left(\begin{array}{cccccc}
6 & 2 & 0 & 0 & 0 & 0 \\
6 & -2 & 0 & 0 & 0 & 0 \\
0 & 0 & -4 & 0 & 0 & 0 \\
-6 u_{y} & -2 u_{y} & 8 u_{x} & -4 & 0 & 0 \\
-6 u_{x} & -2 u_{x} & 8 u_{y} & 0 & -4 & 0 \\
8+6\left(u_{x}^{2}+u_{y}^{2}\right) & 2\left(u_{y}^{2}-u_{x}^{2}\right) & -16 u_{x} u_{y} & 8 u_{y} & 8 u_{x} & 4
\end{array}\right) \\
\\
\left(\begin{array}{c}
k_{3} \\
k_{4} \\
k_{5} \\
k_{6} \\
k_{7} \\
k_{8}
\end{array}\right)=\left(\begin{array}{c}
\kappa_{x x}^{\text {at }}-\kappa_{x x} \\
\kappa_{y y}^{\text {at }}-\kappa_{y y} \\
\kappa_{x y}^{\text {at }}-\kappa_{x y} \\
\kappa_{x x y}^{\text {aty }}-\kappa_{x x y} \\
\kappa_{x y y}^{\text {at }}-\kappa_{x y y} \\
\kappa_{x x y y}^{\text {at }}-\kappa_{x x y y}
\end{array}\right)
\end{gathered}
$$

If we set attractors to equilibrium i.e.

$$
\left(\begin{array}{c}
\kappa_{x x}^{a t} \\
\kappa_{y y}^{a t} \\
\kappa_{x y}^{a t} \\
\kappa_{x x y}^{a t} \\
\kappa_{x y y}^{a t} \\
\kappa_{x x y y}^{a t}
\end{array}\right)=\left(\begin{array}{c}
\rho c_{s}^{2} \\
\rho c_{s}^{2} \\
0 \\
0 \\
0 \\
\rho c_{s}^{4}
\end{array}\right)
$$

and solve the above system, we obtain

$$
\begin{aligned}
k_{3}= & \frac{1}{12 \tau_{3}}\left[\rho\left(u_{x}^{2}+u_{y}^{2}\right)-f_{6}-f_{8}-f_{4}-f_{2}-2\left(f_{5}+f_{3}\right.\right. \\
& \left.\left.+f_{7}+f_{1}-\rho c_{s}^{2}\right)\right] \\
k_{4}= & \frac{1}{4 \tau_{4}}\left[f_{8}+f_{4}-f_{6}-f_{2}+\rho\left(u_{x}^{2}-u_{y}^{2}\right)\right] \\
k_{5}= & \frac{1}{4 \tau_{5}}\left(f_{7}+f_{3}-f_{1}-f_{5}-u_{x} u_{y} \rho\right) \\
k_{6}= & \frac{-1}{\tau_{6}}\left\{\left[f_{5}+f_{3}-f_{7}-f_{1}-2 u_{x}^{2} u_{y} \rho\right.\right. \\
& \left.+u_{y}\left(\rho-f_{8}-f_{4}-f_{0}\right)\right] / 4 \\
& \left.+\frac{u_{x}}{2}\left(f_{7}-f_{1}-f_{5}+f_{3}\right)\right\}+\frac{u_{y}}{2}\left(-3 k_{3}-k_{4}\right)+2 u_{x} k_{5} \\
k_{7}= & \frac{-1}{\tau_{7}}\left\{\left[f_{3}+f_{1}-f_{5}-f_{7}-2 u_{y}^{2} u_{x} \rho\right.\right. \\
& \left.+u_{x}\left(\rho-f_{2}-f_{6}-f_{0}\right)\right] / 4 \\
& \left.+\frac{u_{y}}{2}\left(f_{7}-f_{1}-f_{5}+f_{3}\right)\right\}+\frac{u_{x}}{2}\left(-3 k_{3}+k_{4}\right)+2 u_{y} k_{5} \\
& \frac{1}{4}\left[\frac{1}{\tau_{8}}\left(\rho c_{s}^{4}-\kappa_{x x y y}\right)-8 k_{3}-6 k_{4}\left(u_{x}^{2}+u_{y}^{2}\right)\right. \\
\left.k_{8}\right) & \left.-2 k_{4}\left(u_{y}^{2}-u_{x}^{2}\right)+16 k_{5} u_{x} u_{y}-8 k_{6} u_{y}-8 k_{7} u_{x}\right]
\end{aligned}
$$

for isotropic viscosity we set $\tau_{4}=\tau_{5}=\tau$ and compute $\tau$ from

$$
\nu=\frac{1}{c_{s}}\left(\tau-\frac{1}{2}\right)
$$


More accurate and stable version of CLBM, the Factorized Central Moment LBM was also proposed by Geier [29]. The only difference is in the attractor for the $k_{8}$

$$
\begin{aligned}
k_{8}= & \frac{1}{4}\left[\frac{1}{\tau_{8}}\left(\kappa_{x x y y}^{a t}-\kappa_{x x y y}\right)-8 k_{3}-6 k_{4}\left(u_{x}^{2}+u_{y}^{2}\right)\right. \\
& \left.-2 k_{4}\left(u_{y}^{2}-u_{x}^{2}\right)+16 k_{5} u_{x} u_{y}-8 k_{6} u_{y}-8 k_{7} u_{x}\right]
\end{aligned}
$$

where $\kappa_{x x y y}^{a t}$ is defined as

$$
-\kappa_{x x y y}^{a t}=\kappa_{x x y y}^{e q}=\rho c_{s}^{4} \text { for CLBM }
$$$$
-\kappa_{x x y y}^{a t}=\kappa_{x x}^{*} \kappa_{y y}^{*} \text { for FCM }
$$

and post-collision states $\kappa_{x x}^{*}, \kappa_{y y}^{*}$ are given by

$$
\begin{aligned}
& \kappa_{x x}^{*}=6 k_{3}+2 k_{4}+\kappa_{x x}=6 k_{3}+2 k_{4}+\pi_{x x}-\rho u_{x}^{2} \\
& \kappa_{y y}^{*}=6 k_{3}-2 k_{4}+\kappa_{y y}=6 k_{3}-2 k_{4}+\pi_{y y}-\rho u_{y}^{2}
\end{aligned}
$$

Now we can perform the collision step according to Equation (3)

$$
\mathbf{f}\left(\mathbf{x}_{j}+\mathbf{e}_{i}, t+1\right)=\mathbf{f}+\mathbf{K} \cdot \mathbf{k}
$$

to obtain distribution functions for the next time step.

\subsection{MRT thermal lattice Boltzmann method}

In order to simulate temperature field, the double distribution function model is used i.e. we define second population of distribution functions $g_{i}$ on the D2Q5 lattice. Temperature field is then simulated as a passive scalar obeying the advection-diffusion Equation (2). Associated MRT LBE for $g_{i}$ reads

$g_{i}\left(\mathbf{x}_{j}+\mathbf{e}_{i}, t+1\right)=g_{i}\left(\mathbf{x}_{j}, t\right)-\mathbf{M}^{-1} \mathbf{S}\left(m_{i}\left(\mathbf{x}_{j}, t\right)-m_{i}^{\mathrm{eq}}\left(\mathbf{x}_{j}, t\right)\right)$

with matrices $\mathbf{M}$ and $\mathbf{S}$ defined as [30]

$$
\mathbf{M}=\left(\begin{array}{ccccc}
1 & 1 & 1 & 1 & 1 \\
0 & 1 & 0 & -1 & 0 \\
0 & 0 & 1 & 0 & -1 \\
4 & -1 & -1 & -1 & -1 \\
0 & 1 & -1 & 1 & -1
\end{array}\right) \mathbf{S}=\operatorname{diag}\left(0, \frac{1}{\tau_{\alpha}}, \frac{1}{\tau_{\alpha}}, \frac{1}{\tau_{e}}, \frac{1}{\tau_{\nu}}\right)
$$

and equilibrium moments given by

$$
\mathbf{m}^{\mathrm{eq}}=\left(T, u_{x} T, u_{y} T, a T, 0\right)
$$

to avoid "checkerboard" instabilities [31] we have to keep $a<1$ and thermal diffusivity is then related to $\tau_{\alpha}$ by

$$
\alpha=\frac{4+a}{10}\left(\tau_{\alpha}-\frac{1}{2}\right)
$$

Additional constraints for other relaxation times are [31]

$$
\left(\tau_{e}-\frac{1}{2}\right)=\left(\tau_{\nu}-\frac{1}{2}\right) \quad \text { and } \quad\left(\tau_{\alpha}-\frac{1}{2}\right)\left(\tau_{\nu}-\frac{1}{2}\right)=\Lambda
$$

with $\Lambda$ being the "magical number". Those magical numbers are selected to give special features of the MRT, some of them are [31]
$-\Lambda=\frac{1}{4}$ best stability.

$-\Lambda=\frac{1}{6}$ best diffusion, removes fourth order diffusion error.

$-\Lambda=\frac{1}{12}$ best advection, removes third order advection error.

$-\Lambda=\frac{3}{16}$ exact location of bounce back walls for Poiseuille flow.

In our computations we used $a=2 / 3$ together with $\Lambda=1 / 6$ for solid and $\Lambda=1 / 12$ for fluid lattice sites. Temperature is the only conserved quantity here and is computed as zeroth order moment of $g_{i}$

$$
T=\sum_{i} g_{i}
$$

\subsection{Initial and boundary conditions}

During initialization all lattice sites are setup to the equilibrium with the unit density, zero velocity and prescribed temperature $T_{0}$

$$
f_{i}\left(\mathbf{x}_{j}, 0\right)=\left.f_{i}^{\mathrm{eq}}\right|_{u_{x}=0, u_{y}=0, \rho=1} \quad g_{i}\left(\mathbf{x}_{j}, 0\right)=\left.g_{i}^{\mathrm{eq}}\right|_{T=T_{0}}
$$

Boundary conditions are needed at solid walls, inlets, outlets and symmetry planes. Solid walls are treated with bounce-back boundary conditions located half-way, that results in no-slip conditions for velocity field and adiabatic conditions for the heat transfer

$$
f_{\bar{i}}\left(\mathbf{x}_{b}, t+1\right)=f_{i}^{*}\left(\mathbf{x}_{b}, t\right)
$$

where $\mathbf{x}_{b}$ is boundary adjacent fluid site and $f_{\bar{i}}$ corresponds to $\mathbf{e}_{\bar{i}}=-\mathbf{e}_{i}$. In the case of a non-adiabatic fluidsolid interface no bounce-back is applied to the $g_{i}$ in order to allow heat flowing through this interface. For inlet we use the equilibrium conditions both for velocity and temperature fields i.e.

$$
f_{i}\left(\left(0, N_{y}\right), t\right)=f_{i}^{\mathrm{eq}}\left(\rho_{\mathrm{in}}, \mathbf{u}_{\text {in }}\right) \quad g_{i}\left(\left(0, N_{y}\right), t\right)=g_{i}^{\mathrm{eq}}\left(T_{\text {in }}\right)
$$

Outlet is modelled by first-order extrapolation

$$
f_{i}\left(\left(N_{x}-1, j\right), t+1\right)=f_{i}^{*}\left(\left(N_{x}-2, j\right), t\right) j \in\left[0, N_{y}[i \in 5,6,7\right.
$$

Finally, symmetry boundary conditions are realized through mirroring the populations according to the symmetry axis e.g. symmetry conditions for $x$ axis symmetry are then defined by following relations

$$
f_{i}((j, 0), t+1)=f_{\bar{i}}^{*}((j, 0), t) \quad j \in\left[0, N_{x}[\quad i \in\{1,7,8\}\right.
$$

\subsection{CUDA implementation of the solver}

TLBM is implemented in C language and CUDA [32] framework is used in order to speed up the simulations. Common techniques of code optimization are used. Two copies of lattice's distribution functions are held in GPU memory and accessed alternately i.e. only one kernel performing both collision and streaming step is needed. Data 
Table 1. Average performance of the different kernels on GTX GPUs (in MLUPS).

\begin{tabular}{ccc}
\hline kernel type & Titan Z & Titan \\
\hline Isothermal (FCMLBM) sp & 2410 & 2162 \\
Thermal (FCMLBM/MRT) sp & 1281 & 1265 \\
Isothermal (FCMLBM) dp & 1421 & 1351 \\
Thermal (FCMLBM/MRT) dp & 918 & 865 \\
\hline
\end{tabular}

are kept within structures of arrays to minimize GPU memory latency by minimizing non-coalesced access, together with pull algorithm implementation considering fact that the fastest way to perform streaming on the latest GPUs consists of the uncoalesced read (the streaming step) and the coalesced write (i.e. saving the postcollision state to the second copy of the lattice). Our CUDA implementation techniques are very similar to that published e.g. in reference [8]. GPUs used in computations were GTX GeForce Titan and GTX GeForce Ti$\tan \mathrm{Z}$ with enabled double-precision option. Average performance of single- and double-precisions versions of the kernels for benchmark simulations are summarized in Table 1. Benchmark simulation consists of simple thermal or iso-thermal flow in channel with symmetry boundaries on the top and the bottom of the channel. The main limiting factor of the kernel's performance is not the execution of the kernel's code but the memory transfers within GPU. It was experimentally found that block size of 64 gave best performance in case of single-precision kernels and 128 in case of double-precision kernels. Performance is standardly measured in MLUPS (Million Lattice Sites Updates per Second). Better performance of Titan Z is given by more CUDA cores available (2880 compared to 2688 in the case of Titan).

\section{Simulated problems}

Presented TLBM is first validated using data from the rock bed thermal storage experiment, next thermal characteristic of the fixed bed in a shaft furnace consisting of particles with different size is numerically examined. Plots of thermal response of such bed for two air mass flow rates are presented. In this section experimental setup is described together with model parameters.

\subsection{Rock bed thermal storage experiment}

High temperature thermal storage consisting of the rock bed with hot air as a charging medium will be studied numerically. Experimental device described in references $[33,34]$ consists of the vertical, tubular and thermally insulated vessel filled with spherical particles. The diameter of the vessel is $0.148 \mathrm{~m}$ and the height is $1.2 \mathrm{~m}$. Particles are mono-sized, vertically oriented, densely packed and made of steatite (magnesium silicate) with diameter of $0.02 \mathrm{~m}$. Inlet air temperature is $T_{\text {in }}=823 \mathrm{~K}$ and initially the bed has temperature

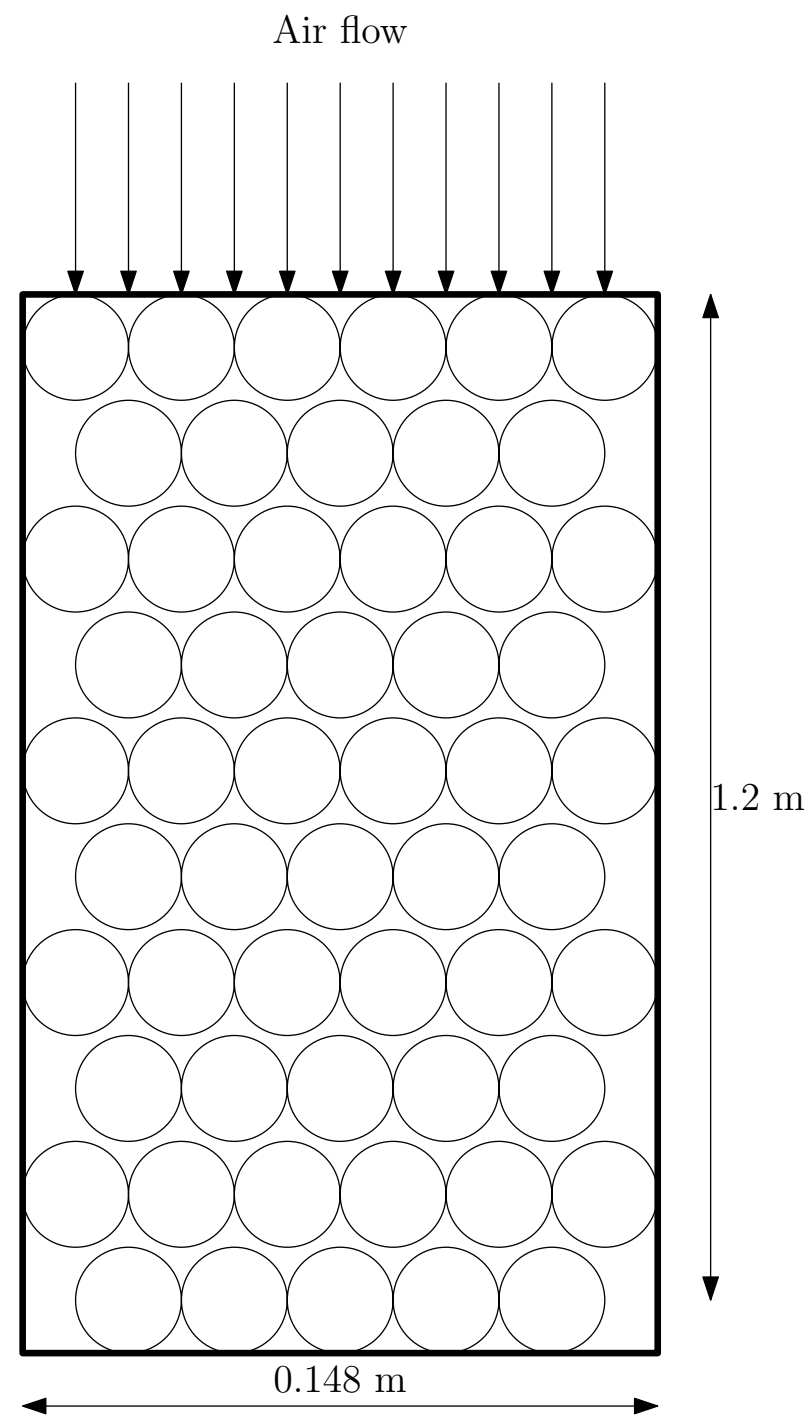

Fig. 2. Vessel geometry with spherical rock bed inside.

$T_{0}=293 \mathrm{~K}$. Air mass flow rate per unit cross section is $\dot{G}=0.225 \mathrm{~kg} \cdot \mathrm{m}^{-2}$.s. Porosity of the bed is $\epsilon=0.4$. The heat diffusivity and kinematic viscosity of the air entering the bed are made linearly temperature dependent in the range of $293 \mathrm{~K}$ and $823 \mathrm{~K}$, solid heat diffusivity is fixed and equal to $\alpha_{s}=8.726 \times 10^{-7} \mathrm{~m}^{2} . \mathrm{s}^{-1}$ and ratio of air heat diffusivity and solid heat diffusivity is around 150 . Reynolds number of the air flow based on inlet velocity and particle diameter is $\operatorname{Re}=42$ and Prandtl number is $\operatorname{Pr}=0.705$, these dimensionless numbers are used to calculate LBM parameters. Physical parameters and appropriate LBM parameters are summarized in Table 2. Geometry setup of the vessel and rock bed is depicted in Figure 2. Part of lattice with indicated solid and fluid nodes are presented in Figure 3. For simulation we used only half part of the bed i.e. cut of $1.2 \mathrm{~m}$ by $0.074 \mathrm{~m}$, with the symmetry boundary condition on the bottom and no-slip adiabatic wall at the top. 
Table 2. Parameters of thermal storage experiment in physical and lattice units (denoted by "+").

\begin{tabular}{cccc}
\hline Physical units & \multicolumn{3}{c}{ Lattice or no units } \\
\hline$T_{\text {in }}$ & $823 \mathrm{~K}$ & $T_{\text {in }}^{+}$ & 1.0 \\
$T_{0}$ & $293 \mathrm{~K}$ & $T_{0}^{+}$ & 0 \\
$H \times D$ & $1.2 \times 0.148 \mathrm{~m}$ & $H^{+} \times D^{+}$ & $1200 \times 74$ \\
$d$ & $0.02 \mathrm{~m}$ & $d^{+}$ & 20 \\
$\dot{G}$ & $0.225 \mathrm{~kg} \cdot \mathrm{m}^{-2} \cdot \mathrm{s}^{-1}$ & $\mathrm{Re}$ & 42 \\
$u_{\text {in }}$ & $0.187 \mathrm{~m} . \mathrm{s}^{-1}$ & $u^{+}$ & 0.12 \\
$\epsilon$ & 0.4 & $\mathrm{Pe}$ & 30 \\
$\alpha_{f}$ & $1.249 \times 10^{-4} \mathrm{~m}^{2} \cdot \mathrm{s}^{-1}$ & $\alpha_{f}^{+}$ & 0.08015 \\
$\nu$ & $8.81 \times 10^{-5} \mathrm{~m}^{2} \cdot \mathrm{s}^{-1}$ & $\nu^{+}$ & 0.056536 \\
$\frac{\alpha_{f}}{\alpha_{s}}$ & $\sim 150$ & $\operatorname{Pr}$ & 0.705 \\
\hline
\end{tabular}

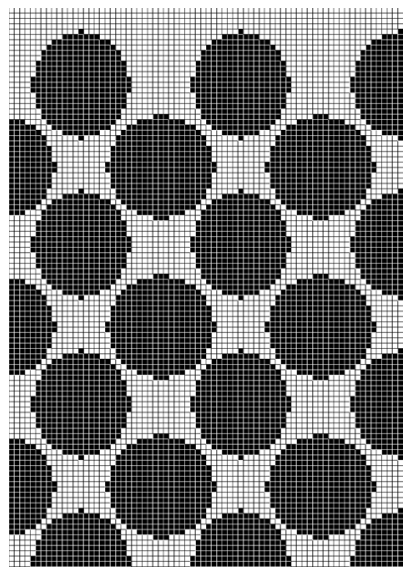

Fig. 3. Detail of lattice (thin grid) structure with fluid (white) and solid (black) sites.

\subsection{Conjugate heat transfer in packed bed}

The large-scale fixed bed inside the shaft furnace with height of $5.12 \mathrm{~m}$ and diameter equal to $2.56 \mathrm{~m}$ is filled up to $3 / 4$ with double-sized spherical and non-spherical particles (see Fig. 4) with diameters $0.21 \mathrm{~m}$ and $0.11 \mathrm{~m}$, irregularly packed with porosity equal to $\epsilon=0.541$. Hot air is blown inside at one side of the bed and leaving on the other like in the previous problem. Two different inlet velocities are considered $u_{1, \text { in }}=0.0601 \mathrm{~m} . \mathrm{s}^{-1}$ and $u_{2, \text { in }}=0.1203 \mathrm{~m} . \mathrm{s}^{-1}$ corresponding to the air mass flows $\dot{M}_{1}=0.4 \mathrm{~kg} \cdot \mathrm{s}^{-1}$ and $\dot{M}_{2}=0.8 \mathrm{~kg} \cdot \mathrm{s}^{-1}$ at normal conditions. Other parameters like viscosity and thermal diffusivities are kept same as in the previous simulation and are summarized together with other parameters in Table 3 .

\section{Results and discussion}

Two numerical experiments were conducted in order to test and validate presented TLBM for use in simulations of conjugate heat transfer and fluid flow in fixed

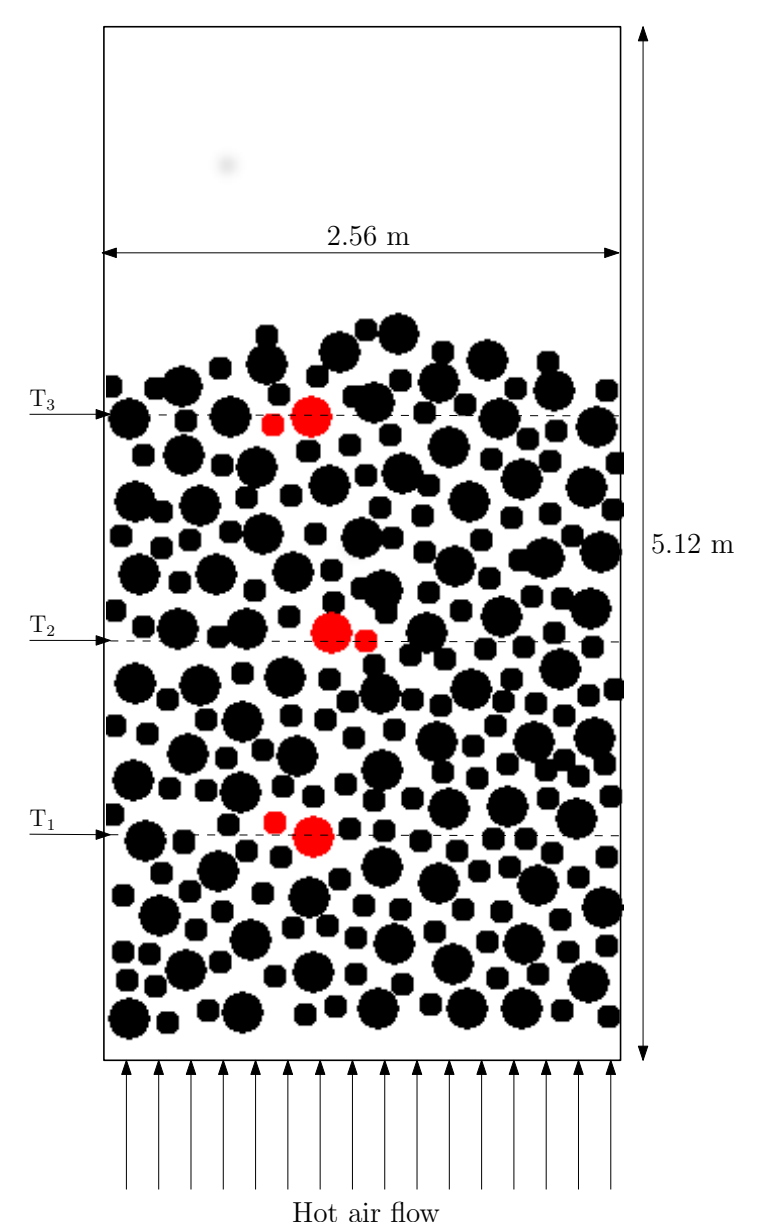

Fig. 4. Geometry setup of the second problem. Fixed bed of two different irregularly packed particles with free space above the bed. Positions where temperature was measured are denoted by arrows and numbers. Marked particles are used for temperature history plots.

beds. In what follow the main results together with discussions provided.

Area averaged bed temperatures along vertical direction starting from the top are presented in Figure 5. 
R. Straka: Mechanics \& Industry 17, 203 (2016)

Table 3. Parameters used in packed bed conjugate heat transfer simulation in physical and lattice units (denoted by "+").

\begin{tabular}{cccc}
\hline Physical units & \multicolumn{3}{c}{ Lattice or no units } \\
\hline$T_{\text {in }}$ & $823 \mathrm{~K}$ & $T_{\text {in }}^{+}$ & 1.0 \\
$T_{0}$ & $293 \mathrm{~K}$ & $T_{0}^{+}$ & 0 \\
$H \times D$ & $5.12 \times 2.56 \mathrm{~m}$ & $H^{+} \times D^{+}$ & $512 \times 256$ \\
$d$ & $0.21 \mathrm{~m}$ & $d^{+}$ & 21 \\
$\dot{M}_{1}$ & $0.4 \mathrm{~kg} \cdot \mathrm{s}^{-1}$ & $\mathrm{Pe}_{1}$ & 101 \\
$u_{1, \text { in }}$ & $0.0601 \mathrm{~m} \cdot \mathrm{s}^{-1}$ & $\mathrm{Re}_{1}$ & 143 \\
$\dot{M}_{2} 1$ & $0.8 \mathrm{~kg} \cdot \mathrm{s}^{-1}$ & $\mathrm{Pe}_{2}$ & 202 \\
$u_{1, \text { in }}$ & $0.1203 \mathrm{~m} \cdot \mathrm{s}^{-1}$ & $\operatorname{Re}_{2}$ & 287 \\
$\epsilon$ & 0.54 & $u^{+}$ & 0.1 \\
$\alpha_{f}$ & $1.249 \times 10^{-4} \mathrm{~m}^{2} . \mathrm{s}^{-1}$ & $\alpha_{1, f}^{+}$ & 0.02087 \\
& & $\alpha_{2, f}^{+}$ & 0.01038 \\
$\nu$ & $8.81 \times 10^{-5} \mathrm{~m}^{2} . \mathrm{s}^{-1}$ & $\nu_{1}^{+}$ & 0.007324 \\
& & $\nu_{2}^{+}$ & 0.014659 \\
$\alpha_{f}$ & $\sim 150$ & $\operatorname{Pr}$ & 0.705 \\
\hline$\alpha_{s}$ & & &
\end{tabular}

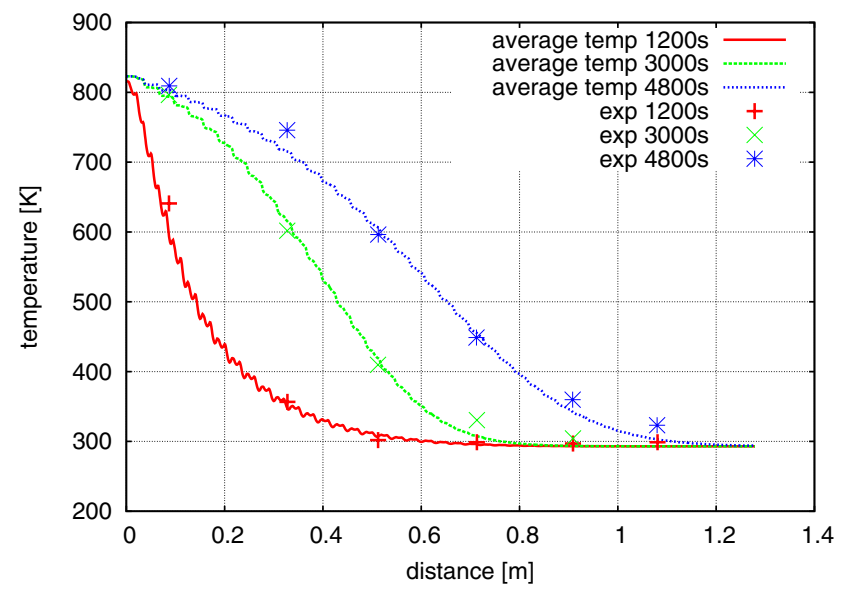

Fig. 5. Validation of the TLBM model using experimental data [34] for high temperature thermal storage vessel. Measured temperatures for three time levels are marked with symbols, averaged computed temperature profiles are plotted as dashed lines.

Solid lines are computed temperature values and symbols denotes experimental values obtained from the literature [34]. Three different time levels are compared. Overall trend of measured temperature profiles is in very good agreement with experimental values. Presented model tends to under predict the temperature for later times and lower half of the bed (note that we measure the distance from the top of the vessel). Possible reason of this is variable heat diffusivity of the bed material with temperature, although in model is kept fixed. Another possible source of errors are inaccuracies in temperature measurements during experiment and errors made during digital processing of experimental data from [34]. Similar results were also obtained in reference [34] with 1D FDM model consisting of two energy conservation equations for fluid and solid phase. Based on the results we can say that

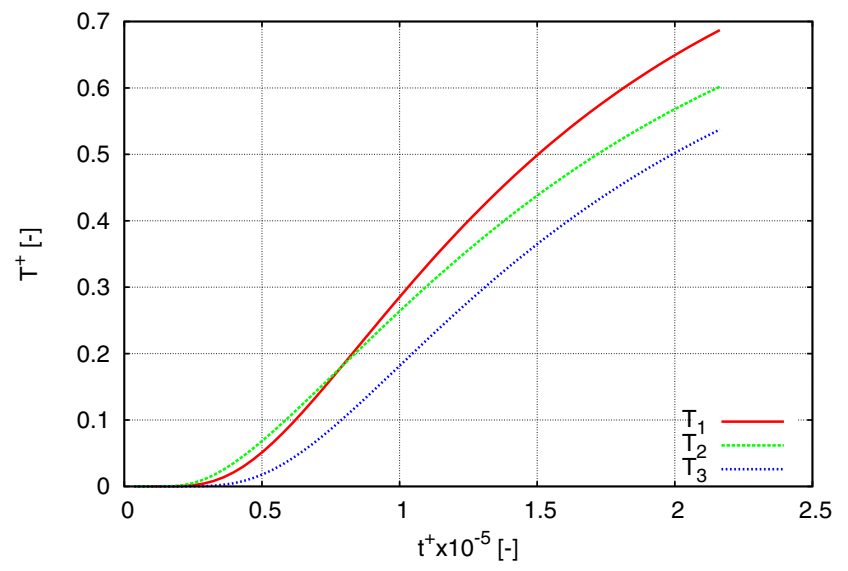

Fig. 6. Temperature history of bigger particles at three different positions. Air mass rate $\dot{M}=0.4 \mathrm{~kg} . \mathrm{s}^{-1}$. Temperature and time are in lattice units.

model is able to correctly predict conjugate heat flow in fixed packed beds. In Table 4 computed and measured values with absolute and relative errors for three time levels are given. Maximal relative error is less than $7 \%$ and tendency of temperature under prediction by the model is clearly visible.

Second numerical experiment of conjugate heat transfer in large-scale fixed bed was performed for two values of hot air mass flow. Temporal profiles of temperature for bigger and smaller particles, together with temperature of air in the vicinity of those particles are depicted in Figures 6-8 for the lower inlet speed and in Figures 911 for higher inlet speed. Temperature was collected near the bed's centerline, in the middle of each particle, which was situated on three different vertical levels $x_{1}=1 \mathrm{~m}$, $x_{2}=2 \mathrm{~m}$ and $x_{3}=3 \mathrm{~m}$. Temperature, velocity magnitude (i.e. $\sqrt{u_{x}^{2}+u_{y}^{2}}$ of real velocity not the superficial one) and 
R. Straka: Mechanics \& Industry 17, 203 (2016)

Table 4. Measured and computed values of average temperature with indicated absolute and relative errors.

\begin{tabular}{cccccc}
\hline $\begin{array}{c}\text { Time } \\
\text { in }[\mathrm{s}]\end{array}$ & $\begin{array}{c}\text { Distance } \\
\text { in }[\mathrm{m}]\end{array}$ & $\begin{array}{c}\text { Measured } \\
\text { temp. in [K] }\end{array}$ & $\begin{array}{c}\text { Computed } \\
\text { temp. in [K] }\end{array}$ & $\begin{array}{c}\text { Absolute } \\
\text { error in [K] }\end{array}$ & $\begin{array}{c}\text { Relative } \\
\text { error in [\%] }\end{array}$ \\
\hline 1200 & 0.087 & 640.854 & 630.489 & -10.364 & -1.617 \\
& 0.328 & 356.707 & 355.466 & -1.242 & -0.348 \\
& 0.512 & 301.829 & 309.388 & 7.559 & 2.504 \\
& 0.713 & 298.780 & 294.912 & -3.869 & -1.295 \\
& 0.909 & 296.951 & 293.085 & -3.866 & -1.302 \\
& 1.081 & 298.780 & 293.004 & -5.777 & -1.933 \\
\hline 3000 & 0.087 & 796.341 & 794.203 & -2.139 & -0.269 \\
& 0.327 & 601.829 & 618.360 & 16.530 & 2.747 \\
& 0.512 & 409.756 & 424.159 & 14.403 & 3.515 \\
& 0.713 & 330.488 & 308.379 & -22.108 & -6.690 \\
& 0.909 & 304.268 & 293.610 & -10.658 & -3.503 \\
& 1.080 & 300.000 & 293.016 & -6.984 & -2.328 \\
\hline 4800 & 0.087 & 809.146 & 803.269 & -5.878 & -0.726 \\
& 0.327 & 745.732 & 716.232 & -29.500 & -3.956 \\
& 0.513 & 596.341 & 610.626 & 14.284 & 2.395 \\
& 0.712 & 448.780 & 457.289 & 8.509 & 1.896 \\
& 0.908 & 359.756 & 345.484 & -14.272 & -3.967 \\
& 1.080 & 323.171 & 303.592 & -19.579 & -6.058 \\
\hline
\end{tabular}

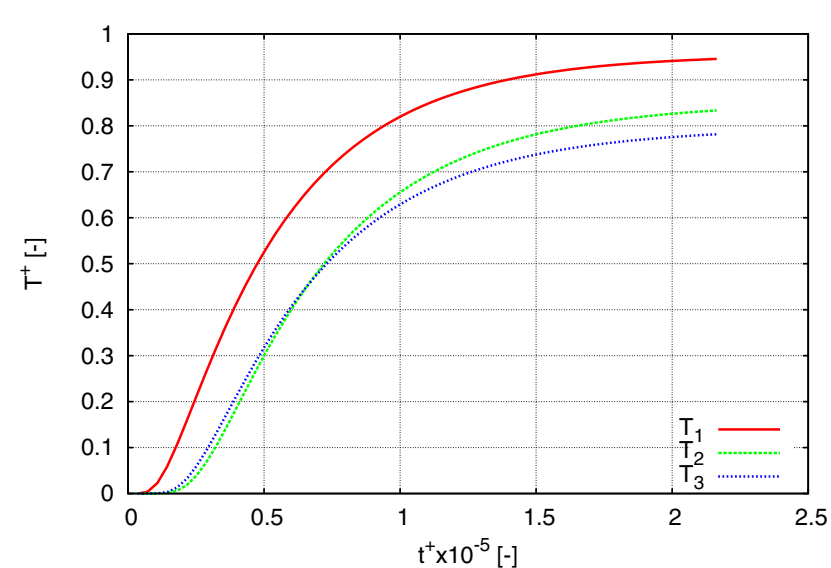

Fig. 7. Temperature history of smaller particles at three different positions. Air mass rate $\dot{M}=0.4 \mathrm{~kg} . \mathrm{s}^{-1}$. Temperature and time are in lattice units.

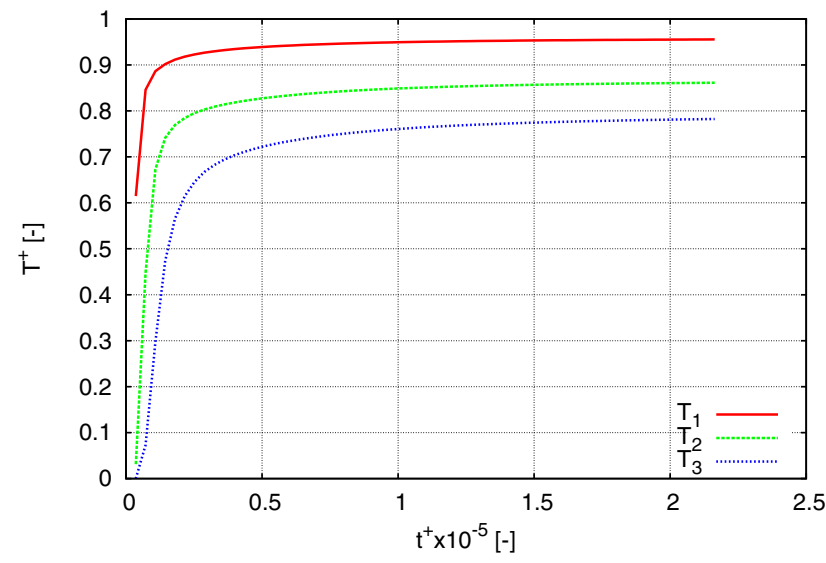

Fig. 8. Temperature history of air at three different positions. Air mass rate $\dot{M}=0.4 \mathrm{~kg} . \mathrm{s}^{-1}$. Temperature and time are in lattice units.

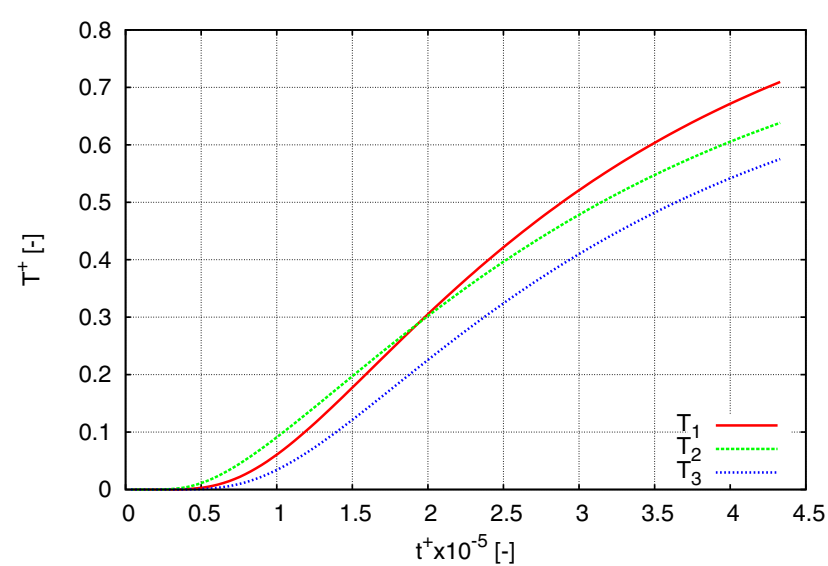

Fig. 9. Temperature history of bigger particles at three different positions. Air mass rate $\dot{M}=0.8 \mathrm{~kg} . \mathrm{s}^{-1}$. Temperature and time are in lattice units.

density (pressure) profiles for the last time step are shown in Figures 12 and 13 in dimensionless lattice units. Effect of channeling is clearly visible in velocity plots. On temperature plots one can recognize slowly heating larger particles with inner temperature gradient indicating high Biot number. From density profiles one can guess pressure distribution and the pressure drop. Temporal behavior of particles exhibits somewhat anomalous heating, when particles closer to inlet are first heated slowly than farther particles (see, e.g. Figs. 6, 7, 9 and 10). This can be explained by channeling effect which causes higher convective heat flux for distant particles due to higher velocity while the temperature of air is still almost the same as at the inlet. Temporal change of air temperatures is first very rapid (as hot air front is passing through the bed) and then slowly increasing (with visible spatial gradient of the air temperature) as particles are being heated up. 


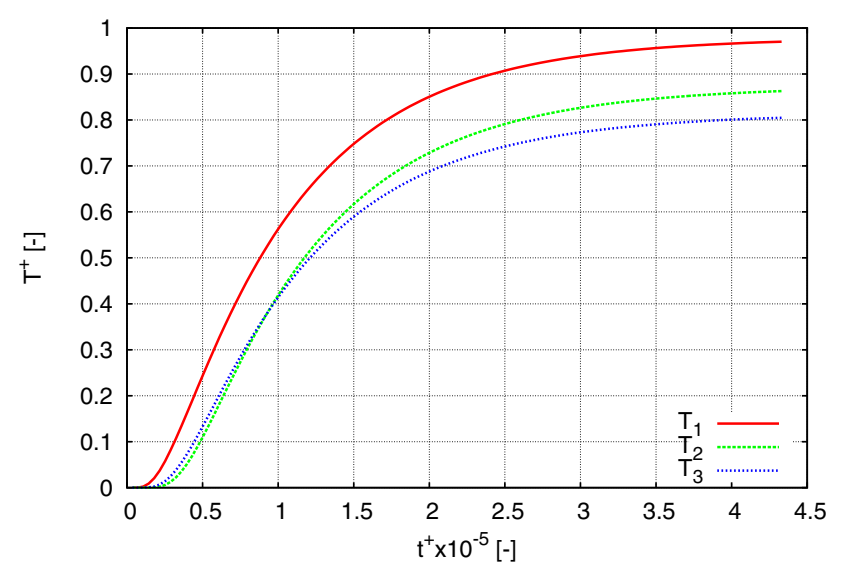

Fig. 10. Temperature history of smaller particles at three different positions. Air mass rate $\dot{M}=0.8 \mathrm{~kg} . \mathrm{s}^{-1}$. Temperature and time are in lattice units.

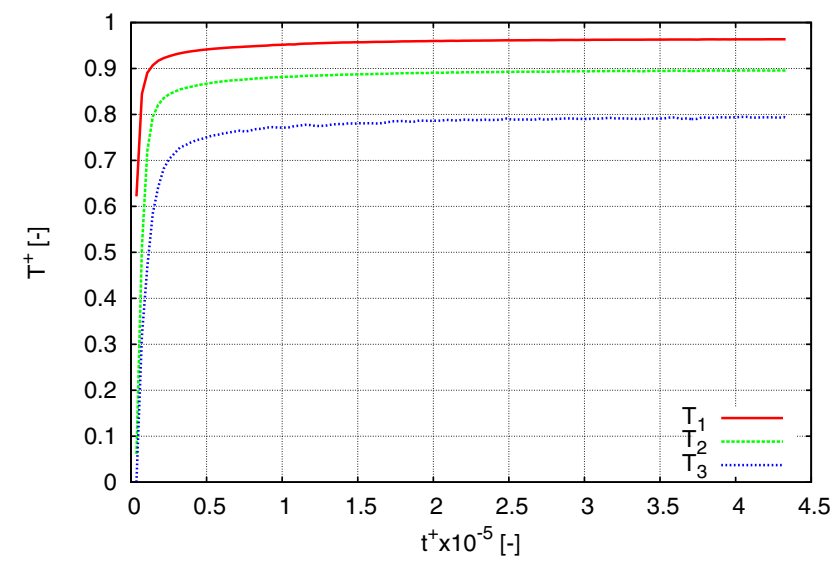

Fig. 11. Temperature history of air at three different positions. Air mass rate $\dot{M}=0.8 \mathrm{~kg} . \mathrm{s}^{-1}$. Temperature and time are in lattice units.

It is quite obvious that smaller particles are heated up faster than bigger ones.

\section{Conclusion}

Thermal lattice Boltzmann model, based on double distribution function populations with factorized central moment method for fluid flow and multiple relaxation time for heat transfer was presented. The validation of the model was based on experimental data from literature [34] on high temperature thermal storage systems employing fixed packed bed of rocks with hot air as a heat transfer medium. The maximal relative error in the comparison was less than $7 \%$ and rather close to $2-3 \%$ with light under prediction of the measured temperatures. Next the thermal characteristics of irregularly packed large scale bed was conducted for packing of two types of spherical particles. Temporal characteristics of six different particles at three different positions together with air temper-

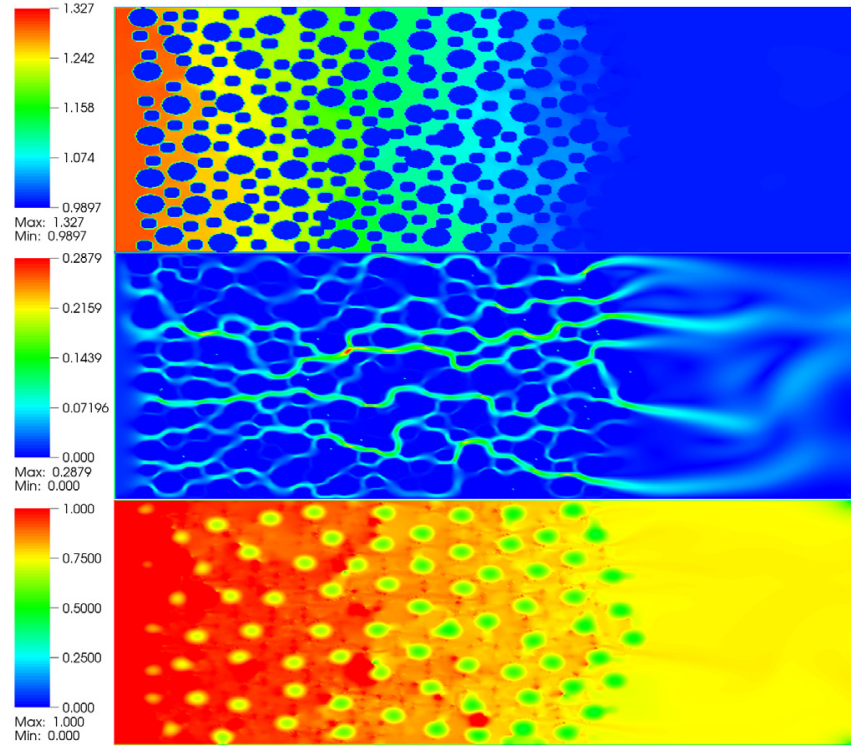

Fig. 12. Instant profiles of density (top), velocity magnitude (middle) and temperature (bottom) in the last step of simulation for $\dot{M}=0.4 \mathrm{~kg} \cdot \mathrm{s}^{-1}$ at the last time step $t^{+}=2.16 \times 10^{5}$ $(t=3600 \mathrm{~s})$. Variables are plotted in lattice units.

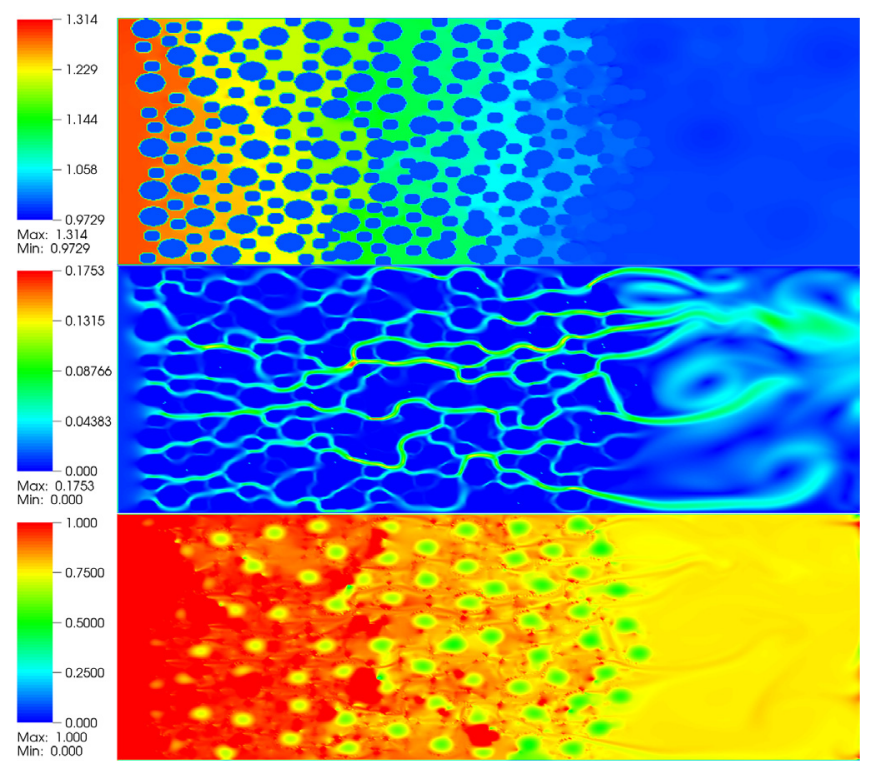

Fig. 13. Instant profiles of density (top), velocity magnitude (middle) and temperature (bottom) in the last step of simulation for $\dot{M}=0.8 \mathrm{~kg} . \mathrm{s}^{-1}$ at the last time step $t^{+}=4.33 \times 10^{5}$ $(t=3600 \mathrm{~s})$. Variables are plotted in lattice units.

ature in the vicinity of those particles were presented. Instant snapshots of density, velocity and temperature fields are shown to reveal channeling effect in the porous structure of the bed.

Acknowledgements. This work has been supported by the research Project No. 15.11.110.239 from the budget for science at Faculty of Metals Engineering and Industrial Computer Science, AGH University of Science and Technology. 


\section{References}

[1] V. Staněk, B.Q. Li, J. Szekely, Mathematical Model of a Cupola Furnace-Part I: Formulation and an Algorithm to Solve the Model, AFS Transactions 100 (1992) 425-437

[2] R. Leth-Miller, A.D. Jensen, P. Glarborg, L.M. Jensen, P.B. Hansen, P. B., S.B. Jørgensen, Investigation of a mineral melting cupola furnace. Part II. Mathematical modeling, Indust. Eng. Chem. Res. 42 (2003) 6880-6892

[3] R. Straka, T. Telejko, 1D Mathematical Model of Coke Combustion, IAENG Int. J. Appl. Math. 45 (2015) 245248

[4] S. Succi, The Lattice Boltzmann Equation for Fluid Dynamics and Beyond, Clarendon, Oxford, 2001

[5] D. Yu, R. Mei, L.S. Luo, W. Shyy, Viscous flow computations with the method of lattice Boltzmann equation, Progress Aerospace Sci. 39 (2003) 329-367

[6] F. Kuznik, C. Obrecht, G. Rusaouen, J.J. Roux, LBM based flow simulation using GPU computing processor, Comput. Math. Appl. 59 (2010) 2380-2392

[7] C. Obrecht, F. Kuznik, B. Tourancheau, J.J. Roux, The TheLMA project: A thermal lattice Boltzmann solver for the GPU, Comput. Fluids 54 (2012) 118-126

[8] N. Delbosc, J.L. Summers, A.I. Khan, N. Kapur, C.J. Noakes, Optimized implementation of the Lattice Boltzmann Method on a graphics processing unit towards real-time fluid simulation, Comput. Math. Appl. 67 (2014) 462-475

[9] M.J. Mawson, A.J. Revell, Memory transfer optimization for a lattice Boltzmann solver on Kepler architecture nVidia GPUs, Comput. Phys. Commun. 185 (2014) 2566-2574

[10] D. d'Humières, I. Ginzburg, M. Krafczyk, P. Lallemand, L.S. Luo, Multiple-relaxation-time lattice Boltzmann models in three dimensions, Philos. Transa. R. Soc. London A 360 (2002) 437-51

[11] S.S. Chikatamarla, C.E. Frouzakis, I.V. Karlin, A.G. Tomboulides, K.B. Boulouchos, Lattice Boltzmann method for direct numerical simulation of turbulent flows, J. Fluid Mech. 656 (2010) 298-308

[12] M.C. Geier, Ab initio derivation of the cascaded lattice Boltzmann automaton, Ph.D. Thesis, University of Freiburg, 2006

[13] C.K. Aidun, J.R. Clausen, Lattice-Boltzmann method for complex flows, Ann. Rev. Fluid Mech. 42 (2010) 439-472

[14] Z. Guo, C. Shu, Lattice Boltzmann method and its applications in engineering (advances in computational fluid dynamics), World Scientific Publishing Company, Singapore, 2013

[15] A.A. Mohamad, Lattice Boltzmann Method, SpringerVerlag, London, 2011

[16] M.C. Sukop, D.T. Thorne Jr., Lattice Boltzmann Modeling: An Introduction for Geoscientists and Engineers, Springer-Verlag, New York, 2006

[17] Y.H. Qian, Simulating thermohydrodynamics with lattice BGK models, J. Sci. Comput. 8 (1993) 231-242
[18] X. Shan, Simulation of Rayleigh-Bénard convection using a lattice Boltzmann method, Phys. Rev. E 55 (1997) $2780-2788$

[19] X. He, S. Chen, G.D. Doolen, A novel thermal model for the lattice Boltzmann method in incompressible limit, J. Comput. Phys. 146 (1998) 282-300

[20] Z. Guo, C. Zheng, B. Shi, T.S. Zhao, Thermal lattice Boltzmann equation for low Mach number flows: decoupling model, Phys. Rev. E 75 (2007) 036704

[21] I.V. Karlin, D. Sichau, S.S. Chikatamarla, Consistent two-population lattice Boltzmann model for thermal flows, Phys. Rev. E 88 (2013) 063310

[22] Z. Malinowski, M. Rywotycki, Modelling of the strand and mold temperature in the continuous steel caster, Arch. Civil Mech. Eng. 9 (2009) 59-73

[23] A. Szajding, T. Telejko, R. Straka, A. Goldasz, Experimental and numerical determination of heat transfer coefficient between oil and outer surface of monometallic tubes finned on both sides with twisted internal longitudinal fins, Int. J. Heat Mass Transfer 58 (2013) 395-401

[24] T. Telejko, A. Szajding, Heat exchange research of the both side finned tubes applied to the heat exchangers, Rynek Energii 5 (2011) 104-110

[25] X. He, L.S. Luo, Theory of the lattice Boltzmann method: from the Boltzmann equation to the lattice Boltzmann equation, Phys. Rev. E 56 (1997) 6811-6817

[26] S. Chen, G.D. Doolen, Lattice Boltzmann method for fluid flows, Ann. Rev. Fluid Mech. 30 (1998) 329-364

[27] D. Hilbert, Mathematical problems, Bull. Am. Math. Soc. 8 (1902) 437-479

[28] X. He, L.S. Luo, Lattice Boltzmann model for the incompressible Navier-Stokes equation, J. Stat. Phys. 88 (1997) 927-944

[29] M.C. Geier, A. Greiner, J.G. Korvink, A factorized central moment lattice Boltzmann method, Eur. Phys. J.Special Topics 171 (2009) 55-61

[30] H. Yoshida, N. Makoto, Multiple-relaxation-time lattice Boltzmann model for the convection and anisotropic diffusion equation, J. Comput. Phys. 229 (2010) 7774-7795

[31] I. Ginzburg, Truncation errors, exact and heuristic stability analysis of two-relaxation-times lattice Boltzmann schemes for anisotropic advection-diffusion equation, Commun. Comput. Phys. 11 (2012) 1439-1502

[32] NVIDIA Corporation, NVIDIA CUDA C Programming Guide v6.5, 2014

[33] A. Meier, C. Winkler, D. Wuillemin, Experiment for modelling high temperature rock bed storage, Solar Energy Mater. 24 (1991) 255-264

[34] M. Hänchen, S. Brückner, A. Steinfeld, High-temperature thermal storage using a packed bed of rocks-heat transfer analysis and experimental validation, Appl. Thermal Eng. 31 (2011) 1798-1806 